\title{
Remarkable Solvent Dependent Excited State Chirality; A Molecular Modulator of Circularly Polarized Luminescence
}

Richard A. van Delden $\dagger$, Nina P. M. Huck $\dagger$, Jacob J. Piet $\ddagger$ John M. Warman $\ddagger$, Stefan C. J. Meskers§, Harry P. J. M. Dekkers§ and Ben L. Feringa $\dagger^{*} *$

$\dagger$ Department of Organic and Molecular Inorganic Chemistry, Groningen Center for Catalysis and Synthesis, University of Groningen, Nijenborgh 4, 9747 AG Groningen, The Netherlands feringa@chem.rug.nl

\section{Supporting Information}

S2 UV-vis absorption of (M)-cis-1 (solid) and (P)-trans-2 (dashed) in benzene.

S3 UV-vis absorption of $(M)$-cis-1 (solid) and ( $P$ )-trans-2 (dashed) in $n$-hexane.

S4 CD spectra of $(M)$-cis-1 (solid) and (P)-trans-2 (dashed) in benzene.

S5 CD spectra of $(M)$-cis-1 (solid) and ( $P$ )-trans-2 (dashed) in $n$-hexane 
UV-vis absorption of $(M)$-cis-1 (solid) and (P)-trans-2 (dashed) in benzene.

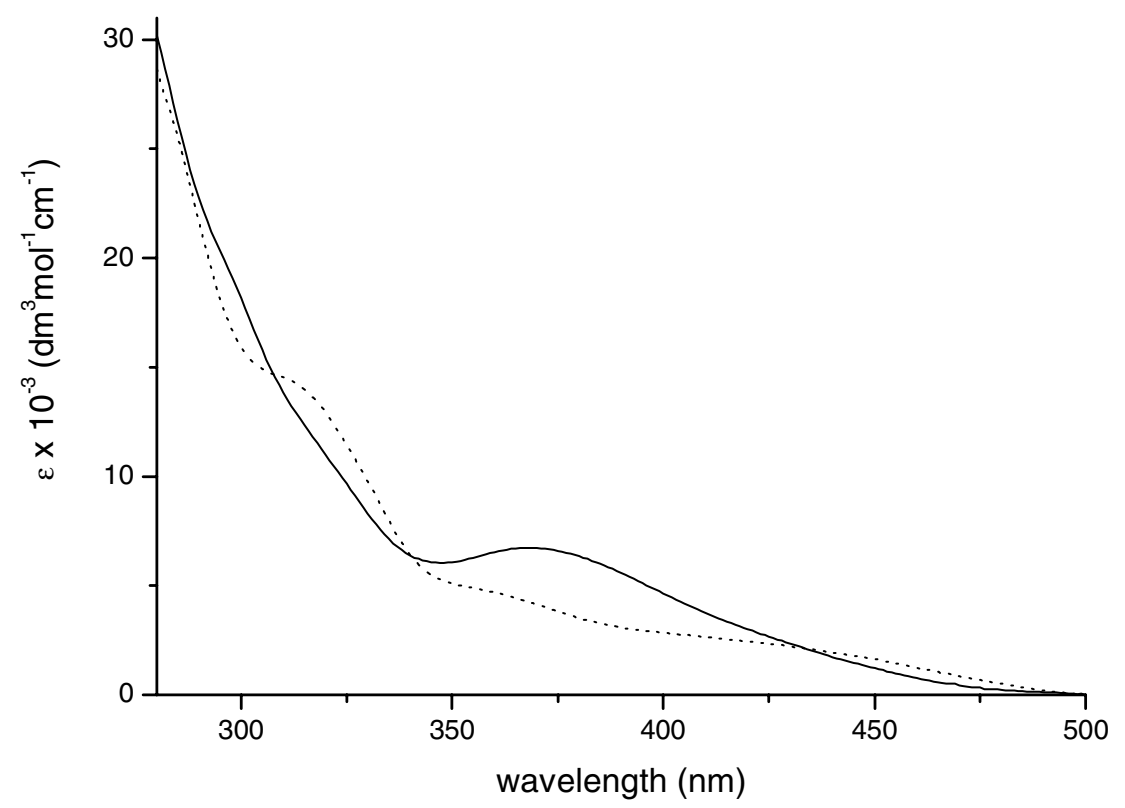


UV/Vis absorption of (M)-cis-1 (solid) and (P)-trans-2 (dashed) in $n$-hexane.

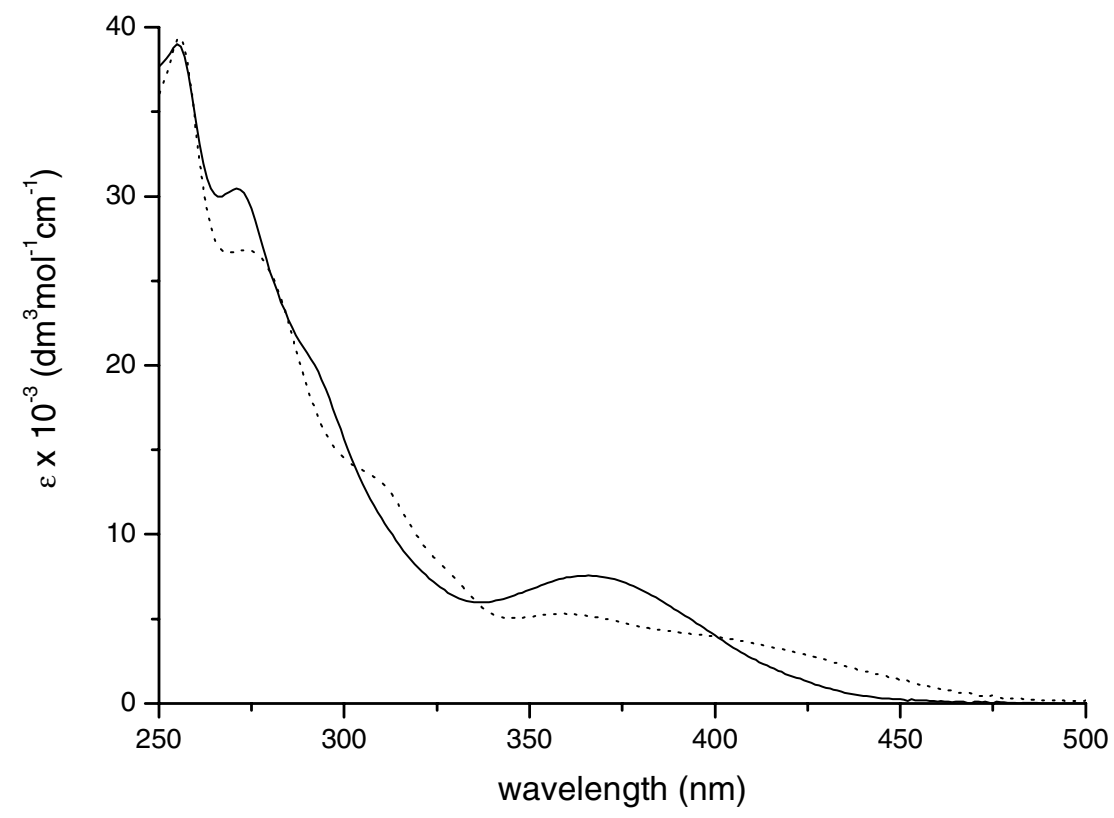


CD spectra of $(M)$-cis-1 (solid) and (P)-trans-2 (dashed) in benzene.

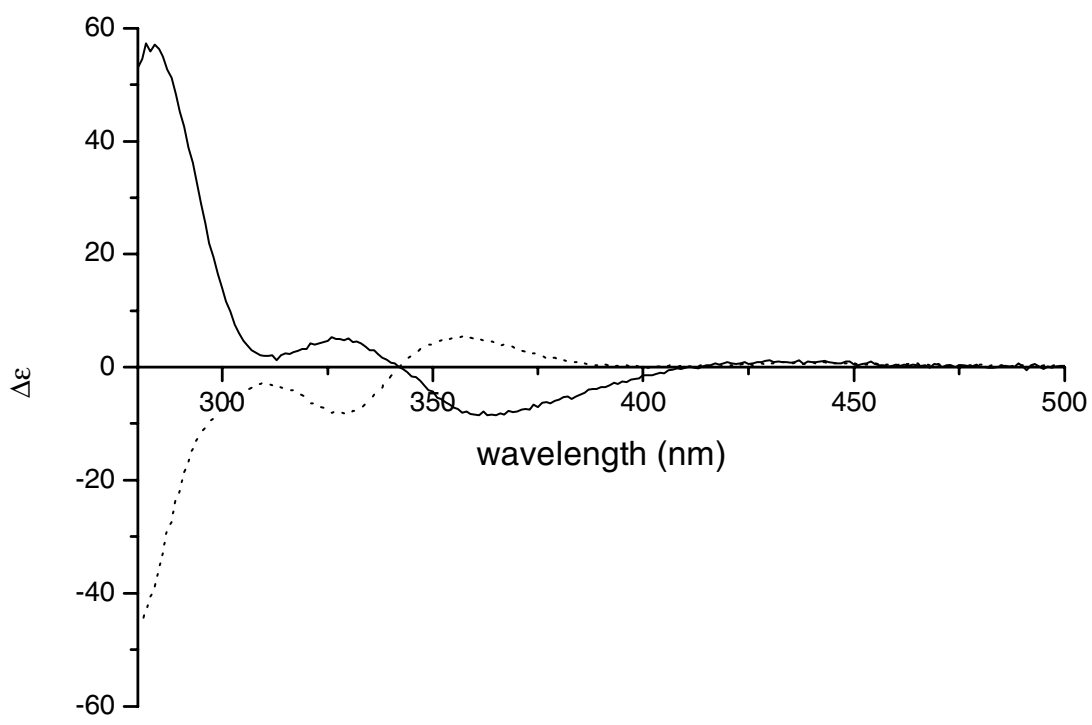


CD spectra of $(M)$-cis-1 (solid) and (P)-trans-2 (dashed) in $n$-hexane

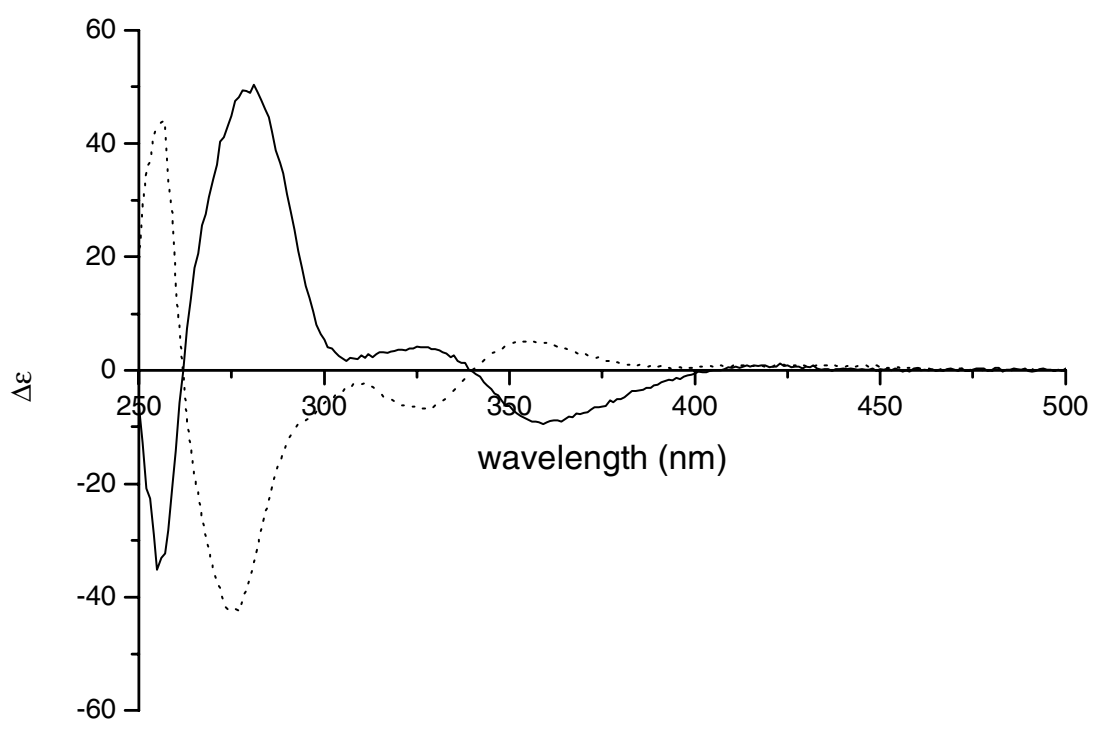

